\title{
The role of TREM2 R47H as a risk factor for Alzheimer's disease, frontotemporal lobar degeneration, amyotrophic lateral sclerosis, and Parkinson's disease
}

Christina M. Lill ${ }^{\mathrm{a}, \mathrm{b}, *}$, Aina Rengmark ${ }^{\mathrm{c}}$, Lasse Pihlstrøm ${ }^{\mathrm{c}}$, Isabella Fogh ${ }^{\mathrm{d}}$, Aleksey Shatunov ${ }^{\mathrm{d}}$, Patrick M. Sleiman ${ }^{\mathrm{e}, \mathrm{f}, \mathrm{g}, 1}$, Li-San Wang ${ }^{\mathrm{h}, 1}$, Tian Liu ${ }^{\mathrm{i}}$, Christina F. Lassen ${ }^{\mathrm{j}}$, Esther Meissner ${ }^{\mathrm{b}}$,

Panos Alexopoulos ${ }^{\mathrm{k}}$, Andrea Calvo ${ }^{1}$, Adriano Chio ${ }^{1, \mathrm{~m}}$, Nil Dizdar ${ }^{\mathrm{n}}$, Frank Faltraco ${ }^{\mathrm{o}}$, Lars Forsgren $^{\mathrm{p}}$, Julia Kirchheiner ${ }^{\mathrm{q}}$, Alexander Kurz ${ }^{\mathrm{k}}$, Jan P. Larsen ${ }^{\mathrm{r}}$, Maria Liebsch ${ }^{\mathrm{b}}$, Jan Linder ${ }^{\mathrm{p}}$, Karen E. Morrison ${ }^{\mathrm{s}, \mathrm{t}}$, Hans Nissbrandt ${ }^{\mathrm{u}}$, Markus Otto ${ }^{\mathrm{q}}$, Jens Pahnke ${ }^{\mathrm{v}, \mathrm{w}}$, Amanda Partch ${ }^{\mathrm{h}}$, Gabriella Restagno ${ }^{\mathrm{x}}$, Dan Rujescu ${ }^{\mathrm{y}}$, Cathrin Schnack $^{\mathrm{q}}$, Christopher E. Shaw ${ }^{\mathrm{d}}$, Pamela J. Shaw ${ }^{\mathrm{z}}$, Hayrettin Tumani $^{\mathrm{q}}$, Ole-Bjørn Tysnes ${ }^{\mathrm{aa}, \mathrm{ab}}$, Otto Valladares ${ }^{\mathrm{h}}$, Vincenzo Silani ${ }^{\mathrm{ac}, \mathrm{ad}}{ }^{\text {, }}$ Leonard H. van den Berg ${ }^{\text {ae }}$, Wouter van Rheenen ${ }^{\text {ae }}$, Jan H. Veldink ${ }^{\mathrm{ae}}$, Ulman Lindenberger ${ }^{\mathrm{i}}$, Elisabeth Steinhagen-Thiessen $^{\text {af }}$, SLAGEN Consortium, Stefan Teipel ${ }^{\text {ag,ah }}$, Robert Perneczky ${ }^{\mathrm{k}, a i, a j}$, Hakon Hakonarson ${ }^{\mathrm{e}, \mathrm{f}, \mathrm{g}}$, Harald Hampel ${ }^{\text {ak,al }}$, Christine A. F. von Arnim ${ }^{\mathrm{q}}$, Jørgen H. Olsen ${ }^{\mathrm{j}}$, Vivianna M. Van Deerlin ${ }^{\mathrm{h}, 1}$, Ammar Al-Chalabi ${ }^{\mathrm{d}}$, Mathias Toft $^{\mathrm{c}}$, Beate Ritz ${ }^{\mathrm{am}}$, Lars Bertram ${ }^{\mathrm{a}, \mathrm{b}, \mathrm{ai}}$

\footnotetext{
${ }^{a}$ Platform for Genome Analytics, Institutes of Neurogenetics \& Integrative and Experimental Genomics, University of Lübeck, Lübeck, Germany

${ }^{b}$ Department of Vertebrate Genomics, Max Planck Institute for Molecular Genetics, Berlin, Germany ${ }^{c}$ Department of Neurology, Oslo University Hospital, Oslo, Norway

${ }^{d}$ Department of Clinical Neuroscience, Institute of Psychiatry, King's College London, London, UK

${ }^{e}$ Center for Applied Genomics, Abramson Research Center, The Children's Hospital of Philadelphia, Philadelphia, PA, USA

${ }^{f}$ Division of Human Genetics, Abramson Research Center, The Children's Hospital of Philadelphia, Philadelphia, PA, USA

${ }^{g}$ Department of Pediatrics, Perelman School of Medicine, University of Pennsylvania, Philadelphia, PA, USA

${ }^{h}$ Department of Pathology and Laboratory Medicine, Perelman School of Medicine at the University of Pennsylvania, Philadelphia, PA, USA ${ }^{i}$ Max Planck Institute for Human Development, Berlin, Germany

${ }^{j}$ Institute of Cancer Epidemiology, Danish Cancer Society, Copenhagen, Denmark

${ }^{k}$ Department of Psychiatry and Psychotherapy, Technische Universität München, Munich, Germany

${ }^{l}$ Rita Levi Montalcini Department of Neuroscience, ALS Center, University of Torino, Torino, Italy

${ }^{m}$ Neuroscience Institute of Turin, Turin, Italy

${ }^{n}$ Department of Neurology, Linköping University, Linköping, Sweden

${ }^{\circ}$ Department of Psychiatry, Psychosomatic Medicine and Psychotherapy, Goethe University of Frankfurt, Frankfurt, Germany

${ }^{p}$ Department of Pharmacology and Clinical Neuroscience, Umeå University, Umeå, Sweden

${ }^{q}$ Department of Neurology, University of Ulm, Ulm, Germany

${ }^{r}$ The Norwegian Centre for Movement Disorders, Stavanger University Hospital, Stavanger, Norway

${ }^{s}$ School of Clinical and Experimental Medicine, College of Medical and Dental Sciences, University of Birmingham, Birmingham, UK

${ }^{t}$ Neurosciences Division, University Hospitals Birmingham NHS Foundation Trust, Birmingham, UK

${ }^{u}$ Department of Pharmacology, The Sahlgrenska Academy at University of Gothenburg, Gothenburg, Sweden

${ }^{v}$ Department of Neuro-/Pathology, University of Oslo and Oslo University Hospital, Oslo, Norway

${ }^{w}$ Lübeck Institute of Experimental Dermatology, University of Lübeck, Lübeck, Germany

${ }^{x}$ Department of Clinical Pathology, Molecular Genetics Unit, Azienda Ospedaliera Città della Salute e della Scienza, Torino, Italy ${ }^{y}$ Department of Psychiatry, University of Halle-Wittenberg, Halle, Germany
}

\footnotetext{
${ }^{1}$ On behalf of the International Collaboration for Frontotemporal Lobar Degeneration; see Appendix for full list of contributors.
} 


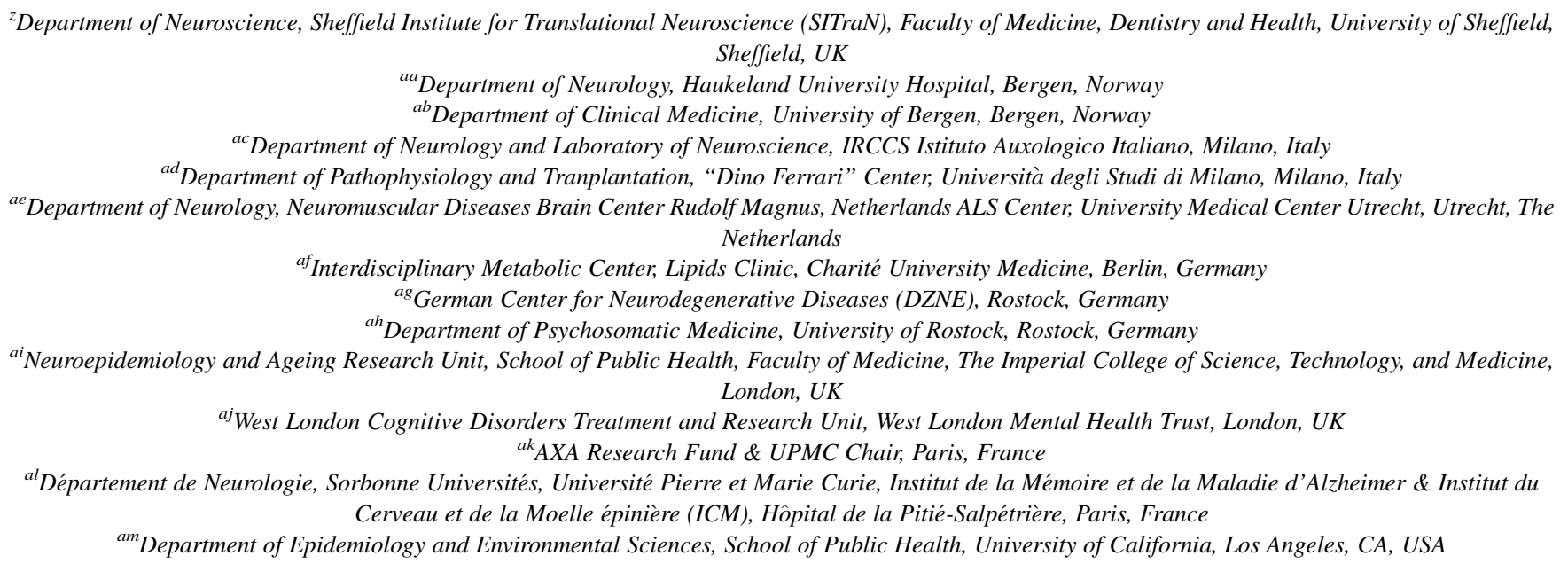

Abstract

A rare variant in TREM2 (p.R47H, rs75932628) was recently reported to increase the risk of Alzheimer's disease (AD) and, subsequently, other neurodegenerative diseases, i.e. frontotemporal lobar degeneration (FTLD), amyotrophic lateral sclerosis (ALS), and Parkinson's disease (PD). Here we comprehensively assessed TREM2 rs75932628 for association with these diseases in a total of 19,940 previously untyped subjects of European descent. These data were combined with those from 28 published data sets by meta-analysis. Furthermore, we tested whether rs75932628 shows association with amyloid beta $\left(\mathrm{A} \beta_{42}\right)$ and total-tau protein levels in the cerebrospinal fluid (CSF) of 828 individuals with AD or mild cognitive impairment. Our data show that rs75932628 is highly significantly associated with the risk of AD across 24,086 AD cases and 148,993 controls of European descent (odds ratio or $\mathrm{OR}=2.71, P=4.67 \times 10^{-25}$ ). No consistent evidence for association was found between this marker and the risk of FTLD $(\mathrm{OR}=2.24, P=.0113$ across 2673 cases $/ 9283$ controls $), \mathrm{PD}(\mathrm{OR}=1.36, P=.0767$ across 8311 cases $/ 79,938$ controls $)$ and ALS $(\mathrm{OR}=1.41, P=.198$ across 5544 cases/7072 controls). Furthermore, carriers of the rs 75932628 risk allele showed significantly increased levels of CSF-total-tau $(P=.0110)$ but not $\mathrm{A} \beta_{42}$ suggesting that TREM2's role in AD may involve tau dysfunction.

(C) 2015 The Alzheimer's Association. Published by Elsevier Inc. All rights reserved.

Keywords: $\quad$ Neurodegenerative disease; Alzheimer disease; Frontotemporal lobar degeneration; Amyotrophic lateral sclerosis; Parkinson disease; TREM2; R47H; rs75932628; Rare variant; Genetic association; GWAS; Imputation; Meta-analysis

\section{Introduction}

Genetic factors substantially contribute to the causation and progression of many-if not all-neurodegenerative diseases. However, for most patients suffering from conditions such as Alzheimer's disease (AD), frontotemporal lobar degeneration (FTLD), amyotrophic lateral sclerosis (ALS), and Parkinson's disease (PD), liability for disease is not the result of causal mutations in single genes, but has a more complex genetic architecture likely resulting from an interplay between multiple genetic and nongenetic risk factors [1]. Genome-wide association studies (GWAS) in $\mathrm{AD}$ and $\mathrm{PD}$ have identified common genetic variants (i.e. single-nucleotide polymorphisms [SNPs]) exerting small to moderate effects in over 50 independent genetic loci $[2,3]$, whereas considerably fewer risk loci have been identified for FTLD and ALS [4,5]. Across all these diseases the established risk variants only explain a fraction of the underlying heritability, i.e., the proportion of phenotypic variance attributable to genetic factors. As a consequence, the focus of many neurodegenerative genetics projects has recently shifted toward investigating rare variants that potentially exert larger effects.

Along these lines, two recent studies have reported on a rare nonsynonymous variant (p.R47H, rs75932628) in TREM2 (encoding the triggering receptor expressed on myeloid cells 2 gene on chromosome 6p21) showing an approximately three- to fivefold increase in $\mathrm{AD}$ risk with a minor allele frequency of $0.12 \%$ to $0.63 \%$ in populations of European descent [6,7]. TREM2 was earlier reported to harbor autosomal-recessive mutations causing NasuHakola disease [8], a neurodegenerative disorder characterized by progressive presenile dementia and cystic bone lesions, and underlying familial FTLD [9]. Given this potentially broader role of TREM2 in neurodegeneration, 
subsequent studies have not only attempted to validate the association between TREM2 rs75932628 and AD risk but have also investigated its potential effects on the risk of other neurodegenerative diseases such as FTLD, ALS, and PD [10-16]. None of these latter studies, however, has thus far yielded conclusive results possibly owing to insufficient power.

The aim of the present project was to assess the overall evidence for association between TREM2 rs75932628 and risk of AD, FTLD, ALS, and PD. To this end, we have generated novel genotype data in a total of 19,940 cases and controls across all four diseases. For each condition, these data were combined with all available previously generated association data by meta-analysis. In addition, we assessed the influence of TREM2 rs75932628 on established cerebrospinal fluid (CSF) biomarkers for AD.

\section{Methods}

\subsection{Subjects}

This study comprised an effective sample size of 20,100 unrelated subjects (i.e., cases of AD, FTLD, ALS, PD, and mild cognitive impairment $[\mathrm{MCI}]$, and predominantly disease-free, elderly controls) of European descent for whom genotyping data on rs75932628 were newly generated by either direct genotyping or extraction from available GWAS microarray data. Subjects and blood samples were ascertained after obtaining written informed consent and appropriate ethical approval at the respective sites. For the $\mathrm{AD}$ analyses, 824 German AD cases were recruited in four specialized centers (LMU and TU Munich, Rostock, and Ulm) all fulfilling the diagnostic criteria of "probable AD" according to the National Institute of Neurological and Communicative Diseases and Stroke-Alzheimer's Disease and Related Disorders Association criteria (NINCDSADRDA) [17] and the American Psychiatric Association's Statistical Manual of Mental Disorders IV (DSM-IV) criteria. The control group for these analyses included a total of 1819 healthy German subjects who did not show clinical signs of dementia or MCI (336 individuals were recruited at the centers in Munich, Rostock, and Ulm, the remaining 1483 individuals were recruited as part of the Berlin Aging Study II, BASE-II [18]). In addition, we carried out quantitative trait locus (QTL) analyses investigating a potential link between rs75932628 and amyloid beta $\left(\mathrm{A} \beta_{42}\right)$ and total tau levels in the CSF of 668 of AD cases and 160 patients diagnosed with MCI (diagnosed based on the criteria by Petersen et al. [19]), which were collected at two of the sites (University Hospital Ulm and LMU Munich).

The FTLD analyses were based on GWAS data from 315 neuropathologically diagnosed FTLD-TDP patients (i.e. the FTLD subtype neuropathologically characterized by transactive response DNA binding protein [TDP]-43 inclusions) of European descent and 1297 Caucasian control individuals recruited from the Children's Hospital of Philadelphia
Health Care Network [4]. FTLD patients were recruited by specialized centers across 11 countries from North America and Europe (United States, Canada, United Kingdom, The Netherlands, Belgium, Spain, Germany, Australia, Finland, France, and Sweden) within the International FTLD Consortium as previously described [4]. Individuals carrying a progranulin mutation $(\mathrm{n}=104)$ or the pathogenic C9ORF72 hexanucleotide expansion $(\mathrm{n}=96)$ were excluded before analysis.

The ALS analyses were based on GWAS data from a total of 4624 cases and 5224 control individuals derived from five previously described data sets of European ancestry that were recruited by the International Consortium on Amyotrophic Lateral Sclerosis Genetics and the Italian Consortium for the Genetics of ALS across three European countries (Italy, The Netherlands, and the UK) [5]. All patients fulfilled the El Escorial revised criteria for ALS [20]. Patients with a family history of ALS or carrying known causative ALS mutations were excluded from the analysis.

For the PD analyses, we directly genotyped rs 75932628 in three independent case-control data sets: the first sample included 1606 PD cases and 1630 controls from Denmark collected as part of a large nationwide population-based study of risk factors for idiopathic PD (Parkinson's disease in Denmark) [21]. The diagnosis was obtained from the Danish National Health Registry and subsequently verified by the review of medical records based on the United Kingdom Parkinson's Disease Society Brain Bank (UKPDSBB) [22] and the Gelb criteria [23]. Additional PD data sets originated from Norway (605 PD cases, 672 controls) and Sweden (774 PD cases, 622 controls) and were collected by five collaborating centers (Oslo, ParkWest, Norway; Gothenburg, Linköping, Umeå, Sweden). PD patients were diagnosed according to the UKPDSBB [22] or the Gelb criteria [23]. All Norwegian and Swedish patients were screened for the LRRK2 G2019S variant (rs34637584), and carriers of the minor allele were excluded from all subsequent association analyses. In addition, control subjects with a history of parkinsonism among firstdegree relatives were excluded from these two data sets.

\subsection{Genotyping}

SNP rs75932628 was genotyped in all samples except for BASE-II, FTLD, and ALS (see below) using allelic discrimination assays based on customized TaqMan assays following the manufacturer's instructions. Each 384-well plate contained approximately four heterozygote sequenceconfirmed control samples genotyped in duplicates across plates. Genotyping and genotype calling were performed blind to phenotypic status. The genotype status of potential carriers of the minor $\mathrm{T}$ allele first determined by allelic discrimination assays was subsequently verified by Sanger sequencing using an ABI3730XL genetic analyzer (Applied Biosystems). Genotypes of rs75932628 for the 1483 BASEII subjects were imputed (using IMPUTE2, http://mathgen. 
stats.ox.ac.uk/impute/impute_v2.html, and the $1000 \mathrm{Ge-}$ nomes Phase 1 CEU reference panel [24]) based on standard quality-controlled GWAS data generated on the GenomeWide Human SNP Array 6.0 (Affymetrix). To increase sensitivity, all samples with a postimputation probability of at least 0.10 for the presence of the minor $\mathrm{T}$ allele of rs75932628 were sequenced as described previously, and sequence-confirmed carrier status of the T allele was hardcoded. The FTLD and ALS case-control samples were genotyped on different Illumina GWAS microarrays and quality-controlled using standard criteria as described earlier $[4,5]$. Imputation of rs75932628 was performed using IMPUTE2 based on the latest 1000 Genomes Project reference panel (build 37/hg19, March 2012; info score for the FTLD data set 0.813 and for the ALS data sets $0.541-0.848)$.

\subsection{CSF biomarker measurements}

CSF samples were collected and processed following standardized protocols and according to international guidelines as previously described [25]. In brief, CSF was sampled in polypropylene tubes after lumbar puncture, centrifuged immediately after collection, and stored within 2 hours at $-80^{\circ} \mathrm{C}$ in Eppendorf tubes until analysis. CSF-A $\beta_{42}$ and total tau protein levels were determined at the collection sites using the commercially available enzyme-linked immunosorbent assays Innotest $\beta$-amyloid[1-42] and hTau Ag kits (Innogenetics) following the manufacturer's instructions. Note that levels for tau phosphorylated at threonine 181 (ptau) were only available in a small subset of patients and thus not included in the analyses of this study.

\subsection{Genetic association analyses}

All genetic association analyses of the newly genotyped $\mathrm{AD}$ and PD data sets were performed in PLINK v1.07 (http://pngu.mgh.harvard.edu/purcell/plink/). Effect sizes and $95 \%$ confidence interval (CI) were estimated based on an additive transmission model with adjustment for age and sex using logistic regression. Significance was assessed using Fisher's exact test and is expressed as two-sided $P$ values. Hardy-Weinberg equilibrium (HWE) was assessed using Pearson's $\chi^{2}$. Rs75932628 was tested for association with ALS and FTLD status using logistic regression analysis based on imputed genotype probabilities as implemented in SNPTEST v2 (https://mathgen.stats.ox.ac.uk/genetics software/snptest/snptest.html). Association results of the ALS data sets were adjusted by specific principal components as described previously [5].

\subsection{Power calculations}

Power was calculated using the Genetic Power Calculator (http://pngu.mgh.harvard.edu/ purcell/gpc/cc2.html) for the detection of an additive odds ratio $(\mathrm{OR})$ of $\sim 3$ (i.e. in the magnitude of effect estimates reported previously for
$\mathrm{AD}[6,7])$ with no between-study heterogeneity based on a risk allele frequency of $0.5 \%$ and assuming a genomewide type 1 error rate of $5 \times 10^{-8}$ or a type- 1 error rate of $1 \times 10^{-4}$ (as proposed previously to represent a "suggestive significance threshold" [26,27], see the "meta-analysis" description in the methods section for details), respectively.

\subsection{Literature screening and meta-analysis}

Genetic association studies investigating the role of TREM2 rs75932628 in AD, FTLD, and PD in populations of European descent were identified and processed using essentially the same strategy as previously described $[28,29]$. In short, this entailed searching the National Center for Biotechnology Information's PubMed database using the keyword "TREM2 [All fields]" (see Supplementary Fig. 1 for an overview of the search and analysis strategy). The titles and/or abstracts of 171 citations published until June 1, 2014 were screened which led to the identification of 26 potentially eligible publications for which the full text was retrieved. Furthermore, we searched for additional relevant association studies by screening cross-references in these publications and review articles on the topic of TREM2 genetics. In addition, one study with relevant data was identified that was in press by June 1, 2014 [30]. Only case-control studies investigating the association of TREM2 rs75932628 and risk for AD, FTLD, ALS, and idiopathic PD that were published in peerreviewed journals in English were considered. Data sets in which rs75932628 was monomorphic in both cases and controls were excluded. Of the 27 potentially eligible publications, 15 studies reporting on 33 independent data sets met our inclusion criteria [6,7,10-16,30-35]. Demographic details and effect size estimates and/or genotype summary data (where available) were extracted from these publications. Published ORs and standard errors were included if they were based on an additive model. If authors provided both unadjusted and adjusted (i.e., for age, sex, APOE 84 [rs429358], and/or population substructure) ORs, the latter were used. Otherwise, genotype summary data were used to calculate the additive OR. Continuity correction was applied to data sets where the minor $\mathrm{T}$ allele of rs75932628 was absent in either cases or controls. In case of insufficient data for inclusion into meta-analyses, authors of the respective publications $[15,33]$ were contacted and missing data were obtained. For data sets overlapping across publications, only the larger data set was retained for meta-analysis (which led to the exclusion of four data sets from three publications $[12,33,34])$. In cases where a putative overlap could not be determined unequivocally, the authors of the respective publications were contacted and the situation clarified (applicable to the eventually confirmed overlap between ref. 12 and 16). Within disease categories, ORs from these publications were combined with those generated as part of our project (see previously) by fixed-effect meta- 
Table 1

Association results for rs75932628 based on newly genotyped data in case-control data sets of AD and PD

\begin{tabular}{|c|c|c|c|c|c|c|c|c|c|c|}
\hline \multirow[b]{2}{*}{ Data set } & \multirow[b]{2}{*}{ Cases ( $\%$ men $)$} & \multirow[b]{2}{*}{ Controls ( $\%$ men $)$} & \multicolumn{2}{|c|}{$\mathrm{AAE}( \pm \mathrm{SD})$} & \multicolumn{2}{|c|}{ Genotypes } & \multicolumn{2}{|c|}{ MAF (\%) } & \multirow[b]{2}{*}{ OR $(95 \% \mathrm{CI})$} & \multirow[b]{2}{*}{$P$} \\
\hline & & & Cases & Controls & Cases & Controls & Cases & Controls & & \\
\hline AD: Germany & $822(37.3)$ & $1818(49.3)$ & $72(8)$ & $67(6)$ & $9 / 813$ & $6 / 1812$ & 0.55 & 0.17 & $5.73(1.80-18.25)$ & .0232 \\
\hline Denmark & $1586(59.8)$ & $1618(60.6)$ & $62(9)$ & $62(10)$ & $5 / 1581$ & $7 / 1611$ & 0.16 & 0.22 & $0.73(0.23-2.30)$ & .774 \\
\hline Norway & $598(63.2)$ & $663(55.7)$ & $66(9)$ & $63(11)$ & $1 / 597$ & $3 / 660$ & 0.08 & 0.23 & $0.37(0.04-3.69)$ & .627 \\
\hline Sweden & $772(60.9)$ & $603(49.6)$ & $69(10)$ & $67(12)$ & $1 / 771$ & $2 / 601$ & 0.07 & 0.17 & $0.37(0.03-4.12)$ & .585 \\
\hline
\end{tabular}

Abbreviations: AAE, age at examination; SD, standard deviation; MAF, minor (T) allele frequency in percent; OR, odds ratio (the reference allele with an OR of 1 corresponds to the major C allele in all settings); CI, confidence interval; AD, Alzheimer's disease; PD, Parkinson's disease.

NOTE. Demographic details of the effective AD and PD data sets and corresponding association results of rs75932628. Effect sizes and 95\% CI were estimated using logistic regression analyses, and $P$ values were calculated using Fisher's exact test. Effect size estimates have been calculated by adjusting for AAE and sex. Data on rs75932628 across the newly genotyped PD data sets ("all new data") were combined using fixed-effect meta-analysis. Genotypes are provided as the number of subjects with the heterozygote T/C genotype/number of subjects with the homozygote C/C genotype. Note that no subject was observed with a homozygote $\mathrm{T} / \mathrm{T}$ genotype.

analyses. All meta-analyses and forest plots were performed using a customized version of the "rmeta" package in R language [29]. The significance threshold in the meta-analysis, i.e., after combining all available data, was defined as $P<5 \times 10^{-8}$ for each meta-analysis, whereas "suggestive evidence" for association on meta-analysis was defined as $P<1 \times 10^{-4}$ as suggested previously [26,27]. Both thresholds take into account the low prior probability of association between any variant in the genome and a phenotype of interest regardless of how many variants were actually tested at the same time [26,27]. Betweenstudy heterogeneity was quantified using the $I^{2}$ metric. Small-study effects (which can be indicative of publication or selective reporting bias) were assessed using Harbord's regression test [36].

\subsection{QTL analyses using CSF biomarkers}

Association between genotypes at rs75932628 and $\log _{10}$-transformed $\mathrm{A} \beta_{42}$ and total tau CSF protein levels was assessed using linear regression as implemented in
PLINK. Analyses were performed with adjustment for age, sex, APOE \&4 (rs429358) genotype status, phenotype (i.e. MCI and AD), and center. We adjusted for phenotype as a potential classical confounder based on the assumption that disease status is correlated with rs75932628 genotype and with $\mathrm{A} \beta_{42}$ and total tau levels. Permuted $P$ values $\left(P_{\text {perm }}\right)$ were calculated based on 1000 rounds of permutation.

\section{Results}

Total genotyping efficiency was $\geq 98.5 \%$ for each of the newly genotyped data sets (see Table 1 for effective sample sizes). Accuracy of genotype calls from the allelic discrimination assays involving the rare allele was $100 \%$ on Sanger sequencing. For each data set, genotypes in controls were distributed according to $\operatorname{HWE}(P \geq .05)$.

In the case-control data sets newly analyzed for this study, the minor $\mathrm{T}$ allele at rs75932628 showed nominally significant association with $\mathrm{AD}$ risk $(\mathrm{OR}=5.73,95 \%$ $\mathrm{CI}=1.80-18.25, P=.0232)$, whereas no significant

Table 2

Association results for rs 75932628 based on imputed GWAS data sets of frontotemporal dementia and ALS

\begin{tabular}{|c|c|c|c|c|c|c|c|c|}
\hline Data sets & Cases & Controls & \multicolumn{2}{|c|}{ Genotypes } & \multicolumn{2}{|l|}{ MAF } & OR $(95 \% \mathrm{CI})$ & $P$ \\
\hline FTLD & 315 & 1297 & $4 / 311$ & $15 / 1282$ & 0.58 & 0.59 & $0.99(0.28-3.47)$ & .990 \\
\hline Netherlands (Utrecht I) & 432 & 420 & $2 / 430$ & $3 / 417$ & 0.28 & 0.32 & $0.82(0.10-6.71)$ & .856 \\
\hline Netherlands (Utrecht II) & 1300 & 1299 & $8 / 1292$ & $10 / 1289$ & 0.32 & 0.40 & $0.76(0.26-2.20)$ & .614 \\
\hline Italy (Chio) & 426 & 226 & $3 / 423$ & $1 / 225$ & 0.39 & 0.19 & $2.84(0.14-57.58)$ & .496 \\
\hline
\end{tabular}

Abbreviations: GWAS, Genome-Wide Association Study; MAF, minor (T) allele frequency in percent; OR, odds ratio (the reference allele with an OR of 1 corresponds to the major C allele in all settings); CI, confidence interval; FTLD, frontotemporal dementia; ALS, amyotrophic lateral sclerosis; SLAGEN, Italian Consortium for the Genetics of ALS.

NOTE. Association results of rs75932628 and FTLD and ALS based on imputed GWAS data sets. Effect sizes were estimated using logistic regression analysis based on genotype probabilities as implemented in SNPTEST v2. Data on rs75932628 across the ALS data sets ("All new data") were combined using fixedeffect meta-analysis. Imputed genotypes are provided as number of subjects with the heterozygote T/C genotype/number of subjects with the homozygote C/C genotype. Note that no subject had an imputed homozygote T/T genotype. 


\section{A}

$$
\text { All datasets }
$$

Dataset specific ORs Current study, Germany Bertram, 2013, USA (GSK) Bertram, 2013, USA, Canada (ADNI) Cuyvers, 2013, Belgium

Ruiz, 2013, Spain

Giraldo, 2013, USA, Europe (TGEN II)

Benitez, 2013, Spain

Pottier, 2013, France

Guerreiro, 2013, USA (Mayo)

Guerreiro, 2013, USA, Europe (ANM, EADI, GERAD)

Guerreiro, 2013, USA, Canada, UK, Portugal

Jonsson, 2013, Germany

Jonsson, 2013, Netherlands (Rotterdam)

Jonsson, 2013, Norway

Jonsson, 2013, USA (eMERGE)

Jonsson, 2013, USA (Emory)

Jonsson, 2013, USA (NIA-LOAD)

Jonsson, 2013, Iceland $\begin{array}{rrr}\text { OR } & 95 \% \mathrm{Cl} \mathrm{I}^{2} \\ 2.71 & {[2.24,3.27]} & 0\end{array}$

$5.73 \quad[1.80,18.25]$

$2.11[0.65,6.86]$

$2.14[0.14,33.22]$

$3.01[0.83,10.93]$

$\begin{array}{ll}3.01 & {[0.83,10.93]}\end{array}$

$2.90[0.14,62.13]$

$15.39[0.87,271.56]$

$4.07[1.12,14.73]$

$6.12[3.12,12.00]$

$2.01 \quad[1.16,3.49]$

$4.52[1.35,15.18]$

$3.15[1.01,9.87]$

$2.45[0.94,6.37]$

$3.52[0.62,19.87]$

3.52 [0.62, 19.87]

4.29 [1.66, 11.07]

.

$\begin{array}{ll}1.93 & {[0.89,4.18]} \\ 2.26 & {[1.71,2.98]}\end{array}$

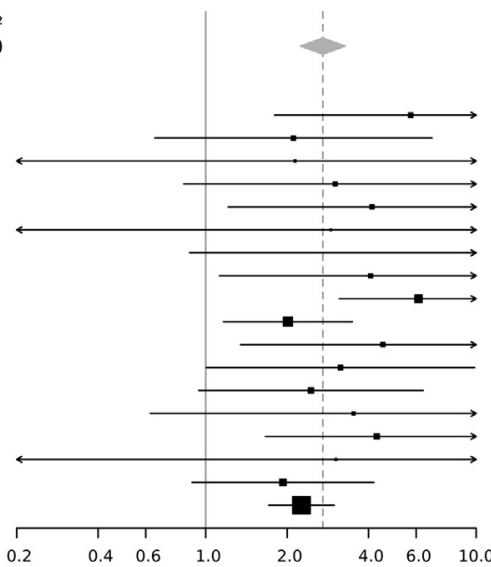

B

All datasets

Dataset specific ORs

Current study, USA, Europe, Australia

Slattery, 2014, UK

Sorroni, 2013, UK

Borroni, 2013, Italy
Cuyvers, 2013, Belgium

Cuyvers, 2013, Bel
Ruiz, 2013, Spain

Rayaprolu, 2013, USA, Canada

OR $\quad 95 \% \mathrm{Cl} \quad \mathrm{I}^{2}$

$2.24[1.20,4.18] 24$

$0.99 \quad[0.28,3.47]$

$0.81[0.13,4.95]$

$2.76[0.09,82.24]$

$3.07[0.62,15.22]$

3.07
$0.53[0.62,15.22]$

$0.06[1.90,13.50]$
0.03

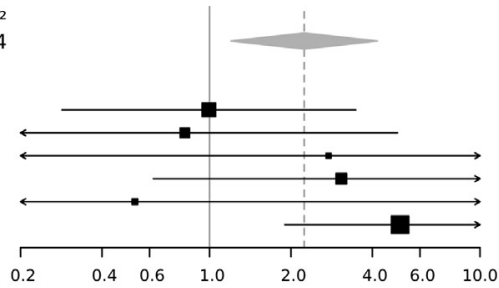

C

All datasets

Dataset specific ORs

Current study, Netherlands (Utrecht I)

Current study, Netherlands (Utrecht II)

Current study, Netherlands

Current study, Italy (Chio)
Current study, Italy (SLAGEN)

Current study, Italy
Current study, UK

Current study, UK
Cady, 2014, USA

(n)

\section{D}

All datasets

Dataset specific ORs Current study, Denmark Current study, Norway Current study, Sweden Jonsson, 2013, Iceland Benitez, 2013, Spain Benitez, 2013, Spain

Rayaprolu, 2013, Ireland Rayaprolu, 2013, Poland Rayaprolu, 2013, USA
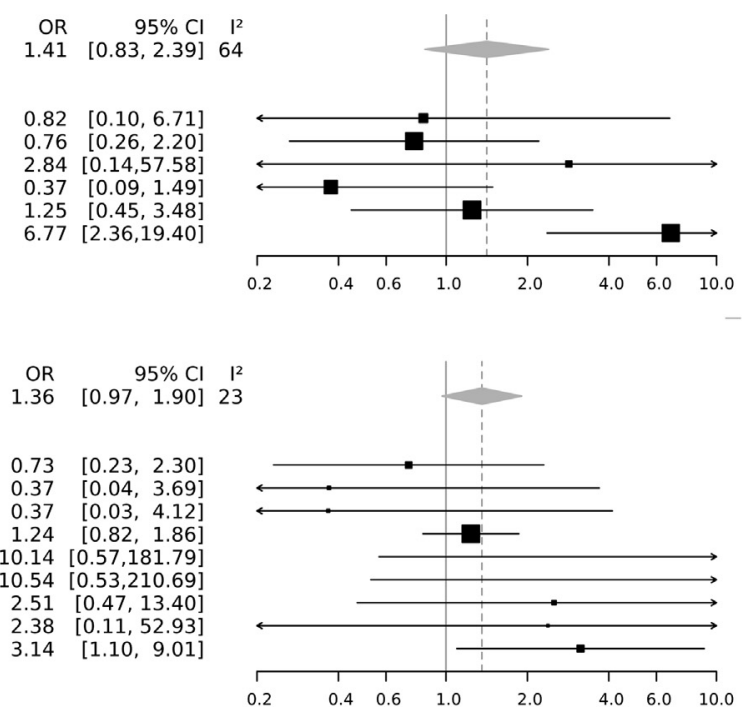

Fig. 1. Meta-analyses of data sets assessing the association between TREM2 rs75932628 and Alzheimer's disease (AD; A), frontotemporal lobar degeneration (FTLD; B), amyotrophic lateral sclerosis (ALS; C), and Parkinson's disease (PD; D). The x-axis depicts the odds ratio (OR). Study-specific ORs (black diamond) and 95\% confidence intervals (CI, lines) were calculated using an additive model. The summary OR and 95\% CI (gray diamonds) were calculated based on fixed-effect meta-analysis. $I^{2}$ is an estimate of the amount of heterogeneity that is beyond chance. Note that effect estimates for data sets "Benitez, 2013, Spain" (A), "Borroni, 2013, Italy", "Ruiz, 2013, Spain" (B), "Benitez, 2013, Spain", "Benitez, 2013, USA", and "Rayaprolu, 2013, Poland" (D) have been calculated after continuity correction to account for zero cell counts. For further details on all data sets and publications included in the respective metaanalyses see Supplementary Table 1.

association was observed with the risk of FTLD, ALS, or PD (Tables 1 and 2). Meta-analysis of rs75932628 across these and all previously published AD data sets (overall comprising $24,086 \mathrm{AD}$ cases and 148,993 controls) revealed genomewide significant association with $\mathrm{AD}$ susceptibility $\left(\mathrm{OR}=2.71,95 \% \mathrm{CI}=2.24-3.28, P=4.67 \times 10^{-25}\right.$; Fig. 1A, Table 3). The meta-analysis across all six FTLD data sets (comprising 2673 FTLD cases and 9283 controls) showed a comparable effect size estimate (OR $=2.24,95 \%$ $\mathrm{CI}=1.20-4.18)$ but only very modest statistical support $(P=.0113$; Fig. 1B, Table 3) and did not surpass the "genome-wide suggestive" significance threshold $\left(P=1 \times 10^{-4}\right)$. Meta-analyses across a total of six independent ALS data sets (comprising 5544 cases and 7072 controls) and nine independent PD data sets (comprising 8311 cases and 79,938 controls) did not provide a convincing statistical 
Table 3

Meta-analysis results of rs75932628 and AD, FTLD, and PD based on newly generated and published data

\begin{tabular}{lcrrrrr}
\hline Disease & Data sets & Cases & Controls & OR (95\% CI & $P$ & $\mathrm{I}^{2}(95 \% \mathrm{CI})$ \\
\hline AD & 18 & 24,086 & 148,993 & $2.71(2.24-3.28)$ & $4.67 \times 10^{-25}$ & $0(0-43)$ \\
FTLD & 6 & 2673 & 9283 & $2.24(1.20-4.18)$ & .0113 & .198 \\
ALS & 6 & 5544 & 7072 & $1.41(0.83-2.39)$ & $64(13-85)$ \\
PD & 9 & 8311 & 79,938 & $1.36(0.97-1.90)$ & .0767 & $23(0-64)$ \\
\hline
\end{tabular}

Abbreviations: OR, odds ratio (the reference allele with an OR of 1 corresponds to the major C allele in all settings); CI, confidence interval; AD, Alzheimer's disease; FTLD, frontotemporal dementia; ALS, amyotrophic lateral sclerosis; PD, Parkinson's disease.

NOTE. Meta-analyses were performed based on a fixed-effect model combing the newly generated data sets and all data sets published until June 1, 2014 that assessed the association of rs75932628 and risk for AD, FTLD, and PD. $\mathrm{I}^{2}$ is the estimate of the amount of between-study heterogeneity that is beyond chance.

support for an effect of rs75932628 on ALS (OR $=1.41,95 \%$ $\mathrm{CI}=0.83-2.39, P=.198$; Fig. $1 \mathrm{C}$, Table 3) or PD risk $(\mathrm{OR}=1.36,95 \% \mathrm{CI}=0.97-1.90, P=.0767$; Fig. $1 \mathrm{D}$, Table 3). The inspection of the respective forest plots (Fig. 1A-D) showed a pronounced risk effect estimate for the initial studies that were not unequivocally replicated in subsequently published data, except for AD. This indicates the presence of a potential "initial study bias" [37]. Accordingly, statistical support for an association of rs75932628 with FTLD, ALS, and PD weakened further on the exclusion of the initial study $\left(P_{\mathrm{FTLD}}=.541, P_{\mathrm{ALS}}=.567, P_{\mathrm{PD}}=.379\right)$. This is in contrast to the $\mathrm{AD}$ replication data, i.e., on exclusion of all data sets used in the two original studies [6,7], which continued to show strong statistical support for an association between $\operatorname{rs75932628}$ and $\mathrm{AD}(P=8.13 \times$ $10^{-07}$ ). Across all meta-analyses only the data for ALS showed evidence for substantial between-study heterogeneity $\left(I^{2}[95 \% \mathrm{CI}]=64\right.$ [13-85], Table 3$)$, which can be attributed to the outlying effect size estimate of the "initial study" of Cady et al. [16] (after removal of this study: $I^{2}[95 \% \mathrm{CI}]=0$ [0-67]). Three data sets subjected to continuity correction showed relatively high ORs (i.e. the AD data set "Benitez, 2013, Spain" [32] and the PD data sets "Benitez, 2013, Spain" and "Benitez, 2013, USA" [14]). Analyses excluding these data sets did not change any of the affected meta-analysis results substantially $\left(\mathrm{OR}_{\mathrm{AD}}=2.69, P=1.33 \times 10^{-24}, \mathrm{OR}_{\mathrm{PD}}=1.28, P=.168\right)$. There was no statistical evidence for the presence of smallstudy effects in any of the four meta-analyses $(P \geq .10)$.

Finally, we performed QTL analyses on the potential correlation between rs75932628 and levels of total tau and $\mathrm{A} \beta 42$, two well-established biomarkers for $\mathrm{AD}$, in CSF samples from 828 German $\mathrm{AD}$ and MCI subjects. As can be seen in Fig. 2, the rs75932628 risk allele (T) showed a modest but statistically significant association with tau in these samples $\left(\beta=0.188, P=.0145, P_{\text {perm }}=.0110\right)$, indicating increased levels of total tau protein in CSF of T allele carriers vs. noncarriers. In contrast, there was no evidence for association between rs75932628 and CSF-A $\beta 42 \quad(\beta=0.058$, $\left.P=.268, P_{\text {perm }}=.295\right)$.

\section{Discussion}

Our study represents the most comprehensive assessment on the potential role of the rare nonsynonymous variant rs75932628 in TREM2 and risk of the four leading neurodegenerative diseases AD, FTLD, PD, and ALS. Our analyses provide strong statistical evidence that the rare nonsynonymous variant rs75932628 in TREM2 substantially increases the risk for AD by approximately 2.7-fold. This OR is larger than that estimated in earlier analyses from our group (OR 1.7) $[33,38]$, which were based on only a small subset of the study samples available. In the first study $\sim 3400$ $\mathrm{AD}$ cases and controls were analyzed [33], whereas a total of $\sim 5300$ subjects were included in the extended and subsequently published analyses [38]. The meta-analysis on rs75932628 presented here, on the other hand, is based on a total of 173,079 AD cases and controls. This vastly increased sample size allows a much more precise estimation of the underlying effect size, especially when dealing with a rare variant such as rs75932628. Furthermore, our findings suggest that the AD risk allele of rs75932628 (T)

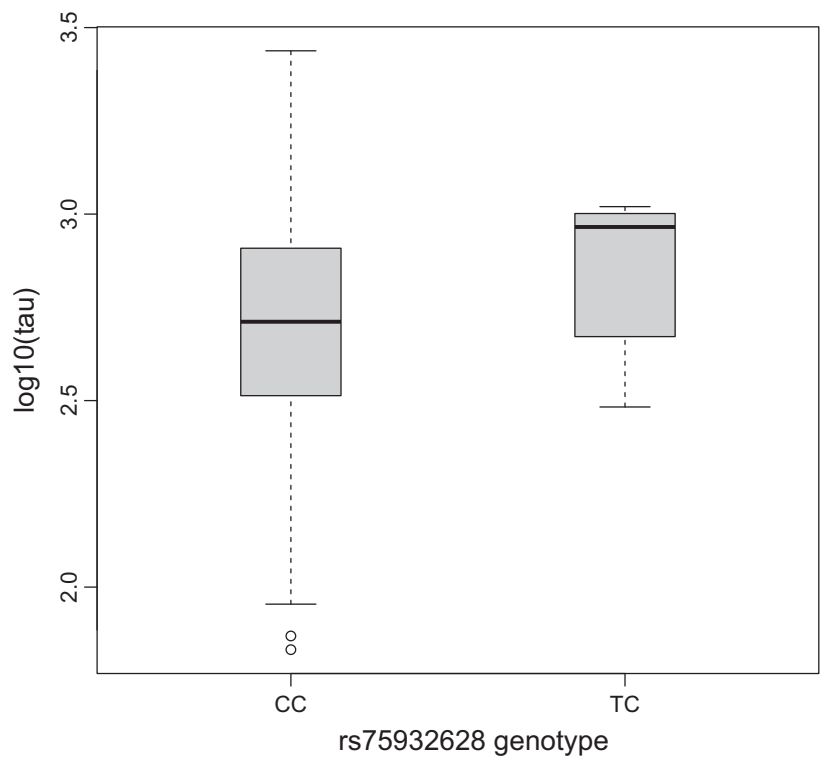

Fig. 2. Box plot of the distribution of total tau protein levels in the cerebrospinal fluid (CSF) dependent on the TREM2 rs75932628 genotype in 828 German patients with Alzheimer's disease and mild cognitive impairment. Horizontal lines represent median values, boxes represent interquartile ranges, and whiskers extend to $1.5 \times$ the interquartile range; values outside this range are depicted as circles. Total tau protein levels (measured in pg/ $\mathrm{ml}$ ) have been log-transformed to the basis 10. Carriers of the TREM2 rs75932628 risk allele (T) showed an increase in total tau protein levels in the CSF. 
is associated with increased levels of CSF total tau but not of $\mathrm{A} \beta_{42}$ in $\mathrm{AD}$ and $\mathrm{MCI}$ patients. This is in line with recently reported data showing a similar effect of rs75932628 on total tau CSF levels on pooling 815 individuals including $\mathrm{AD}$ and MCI patients and controls $\left(\beta=0.19, P=6.9 \times 10^{-4}\right)$ [39]. Collectively, the association between rs75932628 and CSF tau levels may support the previously described interplay of inflammatory response mediated by microglia (i.e., the resident macrophages of the central nervous system, which express TREM2) and pathological processes involving tau [40]. However, despite our comparatively large sample size and the robustness of the linear regression result after permutation, the power of the CSF analyses is limited due to the low minor allele frequency of rs75932628; thus, this finding should be interpreted with caution until established by additional independent data.

In contrast to the highly consistent and significant results observed for $\mathrm{AD}$, our analyses combining new and previously reported association data for FTLD, ALS, and PD currently do not provide convincing statistical support for a role of rs75932628 in contributing to the risk of these diseases, despite having excellent power to detect such effects at genome-wide significant $(>95 \%)$ significance thresholds across all three disease entities. Note, however, that despite the substantial sample sizes accumulated here $(\sim 12,000$ to 88,200 individuals), we cannot reliably exclude the presence of much smaller effect sizes (e.g. ORs $<2$ ) at this time. Power may also be reduced in the presence of clinical heterogeneity within the disease entities examined here; this may in particular apply to the patients diagnosed with FTLD.

Compared with the well-powered risk association analyses, the absence of evidence for an effect of rs75932628 on $\mathrm{A} \beta_{42}$ CSF levels can be attributed to limited power. Of note, in the same data, we observe the well-established association between APOE rs429358 ( $\varepsilon 4)$ and CSF A $\beta_{42}$ levels with strong statistical support (beta $=-0.065$, $\left.P=6.52 \times 10^{-12}, P_{\text {perm }}=.000999\right)$, suggesting that the variance of $\mathrm{A} \beta_{42}$ levels in our $\mathrm{AD} / \mathrm{MCI}$ data set is generally sufficient $\left(\right.$ mean $_{\mathrm{AB} 42 \mathrm{log}}$ : 2.674, standard deviation ${ }_{\mathrm{AB} 42 \mathrm{log}}$ : $0.194)$ to detect genotype-protein level dependencies; the low minor allele frequency of rs75932628 in comparison to APOE rs429358 limits the power of our analysis.

One limitation of our study is that we tested only one polymorphism in TREM2. Thus, we currently do not know whether other variants in this gene do or do not show association with FTLD, ALS, or PD risk. At least in AD, rs75932628 is by far the most strongly and consistently associated SNP in TREM2. Furthermore, we did not have access to sufficiently detailed data for most of the previously published samples to assess potential epistasis between TREM2 rs75932628 and variants in other candidate loci, e.g., AD risk loci. Such an analysis would be particularly interesting for APOE rs429358 (ع4) in the AD data sets analyzed here. Jonsson et al. reported nominal statistical evidence for differential effects of rs75932628 on stratification for APOE rs429358 in their AD discovery cohort, but this was not validated in their replication data sets [6]. Likewise, two other smaller studies did also not find evidence for effect modification of rs75932628 by APOE rs429358 [13,35]. Thus, although the evidence for epistasis between TREM2 rs75932628 and APOE rs429358 in AD is currently not convincing, much larger data sets with subject-level data would be needed to conclusively assess this topic further. Another potential limitation of our study is that ethnic descent was determined based on self-report in most data sets analyzed here, thus the possibility exists that some of our results are affected by subtle population substructure. However, AD data sets adjusting for this potential bias $[6,7,31,33]$ did not show any substantial differences in effect estimates when compared with the data sets using no adjustment (Fig. 1A). Thus, it appears unlikely that the presence of undetected population stratification has had a notable influence on our results. Furthermore, we cannot exclude the potential presence of sampling bias influencing the results for some data sets outside the $\mathrm{AD}$ analyses. This type of bias can occur if patients showing an unclear picture of FTLD, ALS, or PD due to concomitant AD pathology are excluded from the case cohorts, whereas present or potential future $\mathrm{AD}$ cases may remain included in the control cohorts. Inversely, a reduced specificity in the clinical diagnosis of FTLD could lead to contamination of the FTLD cohort with AD cases and thus to an apparent risk effect of rs75932628-T on FTLD that in fact is due to an enrichment of the rare variant in misdiagnosed AD cases. Although the heterogeneity estimate of the FTLD meta-analysis is modest with a large confidence interval (Table 3), the effect estimates of the individual data sets could indeed suggest the presence of some variability (Fig. 1B). However, in this context it is noteworthy that two of the included FLTD data sets included a substantial number of patients with neuropathological diagnosis: 132 of 609 FTLD patients (22\%) reported by Rayaprolu et al [12] were diagnosed neuropathologically. On analyzing this subset only, the authors observed a positive, albeit slightly weaker, effect size estimate than in the overall data set $(\mathrm{OR}=3.56, P=.14$ vs. $5.06, P=.0012)$. This weaker effect size estimate could either represent a chance finding or be at least partially due to a higher specificity of the FTLD diagnosis. At the same time, all patients included in our newly analyzed FTLD data set, which shows a complete null effect for the TREM2 variant $(\mathrm{OR}=0.99, P=.990)$, were diagnosed neuropathologically. In summary, these exploratory considerations do not exclude the possibility that reduced specificity in FTLD diagnosis has affected the genetic association results.

Lastly, we note that the data analyzed here were limited to subjects of European descent. Only limited data are available investigating a potential role of TREM 2 in studies of neurodegenerative diseases in other ethnicities: The allele frequency of TREM2 rs75932628-T appears to be lower in Asian compared with Caucasian populations (e.g., see ref. [41,42]), which further complicates the conclusive assessment across neurodegenerative diseases in Asian 
ethnicities. A recent GWAS on nearly 6000 subjects of African descent reported evidence for association of several SNPs in TREM2 $(P<.009)$ [43], although the authors of this publication did not assess rs75932628 directly. Thus, based on the currently available data, it remains unclear whether TREM2 rs75932628 plays a role as a genetic risk modifier for $\mathrm{AD}$ or other neurodegenerative diseases in populations of non-European descent.

Future studies are needed to elucidate the underlying functional role of TREM2 $\mathrm{R} 47 \mathrm{H}$ (and possibly other disease-associated TREM2 variants) in $\mathrm{AD}$ pathogenesis. Together with the knowledge gained from similar studies on the role of the other established AD risk genes this will shed new light on the pathophysiological processes involved and may ultimately lead to the development of novel strategies for the prevention or treatment of this disease. Furthermore, although the design and execution of targeted clinical trials for $\mathrm{MCI}$ or $\mathrm{AD}$ patients specifically carrying the TREM2 $\mathrm{R} 47 \mathrm{H}$ risk allele may be impractical given the very low frequency (and limited number of available individuals), TREM2 R47H carrier status may still be useful as a variable for stratified analyses in this context similar to what has been proposed for the APOE $\varepsilon 4$ allele [44].

In summary, our study provides compelling evidence that TREM2 rs75932628 is a genetic risk factor for AD. Based on current data, rs75932628 does not appear to play a major role in FTLD, ALS, or PD. Furthermore, individuals affected by $\mathrm{AD}$ or MCI carrying the risk allele show significantly higher CSF tau levels than noncarriers, supporting the notion that TREM2 may exert its pathogenic effects by affecting tau (dys) function.

\section{Acknowledgments}

The authors are grateful to the individuals participating in this study. The authors thank Ms Brit-Maren Schjeide for excellent technical assistance. The authors thank Drs Thorlakur Jonsson and Kari Stefansson for providing us with data of the association analysis of TREM2 rs75932628 and $\mathrm{PD}$ in the Icelandic population beyond those provided in the original publication [15]. The authors thank Dr. Owen A. Ross for providing us with the IDs of the Coriell samples genotyped in the original publication [12].

Competing interests: None of the authors reports any disclosures.

Funding: This project was funded by grants from the German Ministry for Education and Research (BMBF, grant 16SV5538 to L.B., grant 01UW0808 to U.L., and E.S.-T.), the Cure Alzheimer's Fund and Michael J. Fox Foundation (to L.B.), the Innovation Fund of the Max Planck Society (M.FE.A.BILD0002 to U.L.), the Katharina-Hardt-Stiftung, Bad Homburg, Germany, the AXA Research Fund, the Fondation Universite Pierre et Marie Curie and the Fondation pour la Recherche sur Alzheimer, Paris, France (to $\mathrm{HH}$ ), the MND Association UK (to KEM, PS, AAC), the Research Council of Norway and the South-Eastern Norway Regional
Health Authority (to M.T.), the NIEHS of the National Institutes of Health (R01ES013717 to B.R.). Sample collection in Linköping was funded by the European Union as part of the Fifth Framework program (project number QLK4-CT1999-01133 to N.D.). The research leading to these results has received funding from the program "Investissements d'avenir" ANR-10-IAIHU-06.

\section{Supplementary data}

Supplementary data related to this article can be found online at http://dx.doi.org/10.1016/j.jalz.2014.12.009.

\section{RESEARCH IN CONTEXT}

1. Systematic review: Recent studies reported that an amino-acid changing variant (p.R47H) in the TREM2 gene increases the risk for Alzheimer's disease (AD). Our study represents a comprehensive analysis of the potential role of this variant in $A D$ and three other neurodegenerative diseases, i.e. frontotemporal lobar degeneration, amyotrophic lateral sclerosis, and Parkinson's disease. Risk effect estimates of the available case-control datasets (comprising 12,000 to 173,000 individuals per disease) were combined by meta-analysis.

2. Interpretation: Our meta-analyses revealed significant association of $\mathrm{R} 47 \mathrm{H}$ with $\mathrm{AD}$ (increasing risk by 2.7 -fold, $\mathrm{P}=4.7 \times 10-25$ ), but not with the other diseases. Further analyses revealed a significant correlation between $\mathrm{R} 47 \mathrm{H}$ and CSF tau levels, an established AD biomarker.

3. Future directions: Our data suggest that the effects of $\mathrm{R} 47 \mathrm{H}$ are limited to $\mathrm{AD}$ but do not extend to the other neurodegenerative diseases assessed in this study. Functional studies need to clarify a potential role of $\mathrm{R} 47 \mathrm{H}$ in microglia-mediated tau (dys) function.

\section{References}

[1] Lill CM, Bertram L. Towards unveiling the genetics of neurodegenerative diseases. Semin Neurol 2011;31:531-41.

[2] Lambert JC, Ibrahim-Verbaas CA, Harold D, Naj AC, Sims R, Bellenguez C, et al. Meta-analysis of 74,046 individuals identifies 11 new susceptibility loci for Alzheimer's disease. Nat Genet 2013; 45:1452-8.

[3] Nalls MA, Pankratz N, Lill CM, Do CB, Hernandez DG, Saad M, et al. Large scale meta analysis of genome-wide association data in Parkinson's disease reveals 6 novel risk loci. Nat Genet 2014;46:989-93.

[4] Van Deerlin VM, Sleiman PM, Martinez-Lage M, Chen-Plotkin A, Wang LS, Graff-Radford NR, et al. Common variants at 7p21 are associated with frontotemporal lobar degeneration with TDP-43 inclusions. Nat Genet 2010;42:234-9. 
[5] Fogh I, Ratti A, Gellera C, Lin K, Tiloca C, Moskvina V, et al. A genome-wide association meta-analysis identifies a novel locus at $17 q 11.2$ associated with sporadic amyotrophic lateral sclerosis. Hum Mol Genet 2014;23:2220-31.

[6] Jonsson T, Stefansson H, Steinberg S, Jonsdottir I, Jonsson PV, Snaedal J, et al. Variant of TREM2 associated with the risk of Alzheimer's disease. N Engl J Med 2013;368:107-16.

[7] Guerreiro R, Wojtas A, Bras J, Carrasquillo M, Rogaeva E, Majounie E, et al. TREM2 variants in Alzheimer's disease. N Engl J Med 2013;368:117-27.

[8] Paloneva J, Manninen T, Christman G, Hovanes K, Mandelin J, Adolfsson R, et al. Mutations in two genes encoding different subunits of a receptor signaling complex result in an identical disease phenotype. Am J Hum Genet 2002;71:656-62.

[9] Guerreiro RJ, Lohmann E, Brás JM, Gibbs JR, Rohrer JD, Gurunlian N, et al. Using exome sequencing to reveal mutations in TREM2 presenting as a frontotemporal dementia-like syndrome without bone involvement. JAMA Neurol 2013;70:78-84.

[10] Borroni B, Ferrari F, Galimberti D, Nacmias B, Barone C, Bagnoli S, et al. Heterozygous TREM2 mutations in frontotemporal dementia. Neurobiol Aging 2014;35:934.e7-93410.

[11] Cuyvers E, Bettens K, Philtjens S, Van Langenhove T, Gijselinck I, van der Zee J, et al. Investigating the role of rare heterozygous TREM2 variants in Alzheimer's disease and frontotemporal dementia. Neurobiol Aging 2014;35:726.e11-9.

[12] Rayaprolu S, Mullen B, Baker M, Lynch T, Finger E, Seeley WW, et al. TREM2 in neurodegeneration: evidence for association of the p.R $47 \mathrm{H}$ variant with frontotemporal dementia and Parkinson's disease. Mol Neurodegener 2013;8:19.

[13] Ruiz A, Dols-Icardo O, Bullido MJ, Pastor P, Rodríguez-Rodríguez E, López de Munain A, et al. Assessing the role of the TREM2 p.R47H variant as a risk factor for Alzheimer's disease and frontotemporal dementia. Neurobiol Aging 2014;35:444.e1-4.

[14] Benitez BA, Cruchaga C, United States-Spain Parkinson's Disease Research Group. TREM2 and neurodegenerative disease. N Eng1 J Med 2013;369:1567-8.

[15] Jonsson T, Stefansson K. TREM2 and neurodegenerative disease. N Engl J Med 2013;369:1568-9.

[16] Cady J, Koval ED, Benitez BA, Zaidman C, Jockel-Balsarotti J, Allred P, et al. TREM2 variant p.R47H as a risk factor for sporadic amyotrophic lateral sclerosis. JAMA Neurol 2014;71:449-53.

[17] McKhann G, Drachman D, Folstein M, Katzman R, Price D, Stadlan EM. Clinical diagnosis of Alzheimer's disease: report of the NINCDS-ADRDA Work Group under the auspices of Department of Health and Human Services Task Force on Alzheimer's Disease. Neurology 1984;34:939-44.

[18] Bertram L, Böckenhoff A, Demuth I, Düzel S, Eckardt R, Li SC, et al. Cohort profile: The Berlin Aging Study II (BASE-II). Int J Epidemiol 2013;43:703-12.

[19] Petersen RC, Smith GE, Waring SC, Ivnik RJ, Tangalos EG, Kokmen E. Mild cognitive impairment: clinical characterization and outcome. Arch Neurol 1999;56:303-8.

[20] Miller RG, Munsat TL, Swash M, Brooks BR. Consensus guidelines for the design and implementation of clinical trials in ALS. World Federation of Neurology committee on Research. J Neurol Sci 1999; 169:2-12.

[21] Rugbjerg K, Ritz B, Korbo L, Martinussen N, Olsen JH. Risk of Parkinson's disease after hospital contact for head injury: population based case-control study. BMJ 2008;337:a2494.

[22] Hughes AJ, Daniel SE, Kilford L, Lees AJ. Accuracy of clinical diagnosis of idiopathic Parkinson's disease: a clinico-pathological study of 100 cases. J Neurol Neurosurg Psychiatry 1992;55:181-4.

[23] Gelb DJ, Oliver E, Gilman S. Diagnostic criteria for Parkinson disease. Arch Neurol 1999;56:33-9.

[24] Khurana E, Fu Y, Colonna V, Mu XJ, Kang HM, Lappalainen T, et al. Integrative annotation of variants from 1092 humans: application to cancer genomics. Science 2013;342:1235587.
[25] Schjeide BMM, Schnack C, Lambert JC, Lill CM, Kirchheiner J, Tumani H, et al. The role of clusterin, complement receptor 1 , and phosphatidylinositol binding clathrin assembly protein in Alzheimer disease risk and cerebrospinal fluid biomarker levels. Arch Gen Psychiatry 2011;68:207-13.

[26] Sawcer S. Bayes factors in complex genetics. Eur J Hum Genet 2010; $18: 746-50$.

[27] Broer L, Lill CM, Schuur M, Amin N, Roehr JT, Bertram L, et al. Distinguishing true from false positives in genomic studies: p values. Eur J Epidemiol 2013;28:131-8.

[28] Bertram L, McQueen MB, Mullin K, Blacker D, Tanzi RE. Systematic meta-analyses of Alzheimer disease genetic association studies: the AlzGene database. Nat Genet 2007;39:17-23.

[29] Lill CM, Roehr JT, McQueen MB, Kavvoura FK, Bagade S, Schjeide BM, et al. Comprehensive research synopsis and systematic meta-analyses in Parkinson's disease genetics: The PDGene database. PLoS Genet 2012;8:e1002548.

[30] Slattery C, Beck JA, Harper L, Adamson G, Abdi Z, Uphill J, et al. R47H TREM2 variants increases risk of typical early-onset Alzheimer's disease but not of prion or frontotemporal dementia. Alzheimers Dement 2014;10:602-6084.

[31] Giraldo M, Lopera F, Siniard AL, Corneveaux JJ, Schrauwen I, Carvajal J, et al. Variants in triggering receptor expressed on myeloid cells 2 are associated with both behavioral variant frontotemporal lobar degeneration and Alzheimer's disease. Neurobiol Aging 2013;34:2077.e11-8.

[32] Benitez BA, Cooper B, Pastor P, Jin SC, Lorenzo E, Cervantes S, et al. TREM2 is associated with the risk of Alzheimer's disease in Spanish population. Neurobiol Aging 2013;34:1711.e15-7.

[33] Bertram L, Parrado AR, Tanzi RE. TREM2 and neurodegenerative disease. N Engl J Med 2013;369:1565.

[34] Gonzalez Murcia JD, Schmutz C, Munger C, Perkes A, Gustin A, Peterson M, et al. Assessment of TREM2 rs75932628 association with Alzheimer's disease in a population-based sample: the Cache County Study. Neurobiol Aging 2013;34:2889.e11-3.

[35] Pottier C, Wallon D, Rousseau S, Rovelet-Lecrux A, Richard AC, Rollin-Sillaire A, et al. TREM2 R47H variant as a risk factor for early-onset Alzheimer's disease. J Alzheimers Dis 2013;35:45-9.

[36] Harbord RM, Egger M, Sterne JA. A modified test for small-study effects in meta-analyses of controlled trials with binary endpoints. Stat Med 2006;25:3443-57.

[37] Pfeiffer T, Bertram L, Ioannidis JP. Quantifying selective reporting and the Proteus phenomenon for multiple datasets with similar bias. PLoS One 2011;6:e18362.

[38] Hooli BV, Parrado AR, Mullin K, Yip WK, Liu T, Roehr JT, et al. The rare TREM2 R47H variant exerts only a modest effect on Alzheimer disease risk. Neurology 2014;83:1353-8.

[39] Cruchaga C, Kauwe JS, Harari O, Jin SC, Cai Y, Karch CM, et al. GWAS of cerebrospinal fluid tau levels identifies risk variants for Alzheimer's disease. Neuron 2013;78:256-68.

[40] Zilka N, Kazmerova Z, Jadhav S, Neradil P, Madari A, Obetkova D, et al. Who fans the flames of Alzheimer's disease brains? Misfolded tau on the crossroad of neurodegenerative and inflammatory pathways. J Neuroinflammation 2012;9:47.

[41] Yu J-T, Jiang T, Wang YL, Wang HF, Zhang W, Hu N, et al. Triggering receptor expressed on myeloid cells 2 variant is rare in late-onset Alzheimer's disease in Han Chinese individuals. Neurobiol Aging 2014; 35:937.e1-3.

[42] Miyashita A, Wen Y, Kitamura N, Matsubara E, Kawarabayashi T, Shoji M, et al. Lack of genetic association between TREM2 and late-onset Alzheimer's disease in a Japanese population. J Alzheimers Dis 2014;41:1031-8.

[43] Reitz C, Mayeux R, Alzheimer's Disease Genetics Consortium. TREM2 and neurodegenerative disease. N Engl J Med 2013;369:1564-5.

[44] Kennedy RE, Cutter GR, Schneider LS. Effect of APOE genotype status on targeted clinical trials outcomes and efficiency in dementia and mild cognitive impairment resulting from Alzheimer's disease. Alzheimers Dement 2014;10:349-59. 